\title{
Evaluation of Manual Traffic Control in Directing Traffic Operations for Major Special Events: Clemson University Football Game Day Experience
}

\author{
Gowtham Cherukumalli ${ }^{1}$, Wayne Sarasua ${ }^{1,}$, , Stephen Fry ${ }^{1}$, William Davis ${ }^{2}$ \\ ${ }^{1}$ Department of Civil Engineering, Clemson University, Clemson, US \\ ${ }^{2}$ Department of Civil \& Environmental Engineering, Citadel, Charleston, US
}

Email address:

gcheruk@g.clemson.edu (G. Cherukumalli), sarasua@clemson.edu (W. Sarasua), sdfry@g.clemson.edu (S. Fry), jeff.davis@citadel.edu (W. Davis)

${ }^{*}$ Corresponding author

\section{To cite this article:}

Gowtham Cherukumalli, Wayne Sarasua, Stephen Fry, William Davis. Evaluation of Manual Traffic Control in Directing Traffic Operations for Major Special Events: Clemson University Football Game Day Experience. American Journal of Traffic and Transportation Engineering. Vol. 5, No. 2, 2020, pp. 20-28. doi: 10.11648/j.ajtte.20200502.11

Received: September 21, 2018; Accepted: May 31, 2019; Published: May 18, 2020

\begin{abstract}
Crowd ingress and egress occurring before and after large special events results in oversaturation of the traffic network and is a crucial issue event planners and transportation officials must address. Because intersections constitute the greatest restraint in network traffic flows, efficient operation during special event peak flows is crucial. This research focuses on evaluating how well traffic control officers optimize intersection operations during heavily oversaturated conditions associated with large-scale special events. Network traffic data, including volumes, queues, and traffic officer signal/right-ofway phase times, were collected before and after four Clemson University home football games during the 2014 and 2015 season. Actual traffic count volumes were adjusted to account for vehicle queues and used to develop optimal signal timings using Synchro. These results are compared to field-observed intersection operation using officer directed hand-signaling and officer traffic signal pushbutton operation. A microscopic VISSIM model was also created for both manual control and optimized control scenarios to determine average delays for each approach and to improve the interpretation of macroscopic Synchro results. Findings indicate that traffic officers perform well in near saturated conditions; however, optimized signal timings provide reduced approach delay and overall intersection delay in heavily oversaturated conditions. The paper includes recommendations on how traffic officers can improve intersection performance. The paper also describes how results from data collection, analysis, and simulation modeling have been used to make recommendations to law enforcement in evaluating traffic operations and identifying improvements. Intersection control is crucial to the implementation of the overall traffic management plan, and the research findings provide insight to the effectiveness of law enforcement control of intersection operations.
\end{abstract}

Keywords: Special Event Traffic, Pushbutton Operations, Traffic Officer Intersection Control

\section{Introduction}

Extreme traffic congestion may occur during planned special events such as concerts, conventions, fairs, and sporting events. Public and private agencies are constantly being put to the test trying to control the flow of traffic for these events, both safely and efficiently [1]. According to a FHWA report by Latoski, the term planned special event is used to describe these activities because of their known locations, scheduled times of occurrence, and associated operating characteristics [2]. A planned special event creates an increase in travel demand and may require road closures to stage the event. Unlike roadway construction activities or traffic incidents that usually only constrain travel within a single corridor, planned special events affect travel in all corridors serving the event venue.

The campus of Clemson University frequently hosts planned special events throughout the year, with the largest 
events occurring during home football games played on campus at Clemson Memorial Stadium. Football games repeatedly attract large crowds in excess of 80,000 fans, most arriving from out of town via personal vehicle. The Athletic Department of Clemson University works closely with law enforcement agencies to effectively orchestrate traffic control and implement traffic circulation plans to accommodate the ingress and egress of fans attending home football games. For a small town in a rural area, attempting to route traffic flows through the local transportation network, designed for a campus with 20,000 students and a permanent full time population of 13,000 residents, is quite challenging and a crucially important component of successfully executing the overall special event plan. Towards achieving this goal, the Athletic Department engaged faculty and graduate students in Clemson's Civil Engineering Department to study traffic flows, delays, congestion, parking demand, and circulation patterns occurring before and after home football games. This research uses data collected before and after home football games, during the 2014 and 2015 seasons, at 17 critical network intersections, to analyze traffic flow parameters, conduct simulation models, and evaluate special event traffic operations effectiveness.

Planning for special events poses a unique and diverse set of challenges and coordination across multiple agencies that are charged with managing and providing transportation system safety, security, mobility, and reliability. The challenges include: 1) Managing intense peak travel demand, 2) Mitigating potential capacity constraints, 3) Establishing and implementing comprehensive circulation patterns, 4) Influencing utility values associated with various travel mode choices, and 5) Accommodating large pedestrian flows, especially in proximity to the event venue site location.

The current special event traffic management plan for Clemson University's football games attempts to address each of these transportation challenges through coordination across a number of participating agencies. A variety of techniques are used, including short-term establishment of contraflow lanes along strategically located roadways, the use of satellite parking lots, combined with shuttle bus service, and the extensive use of law enforcement officers at major intersections and other crucial locations. Law enforcement traffic control officers typically manage rightof-ways at major intersections, either entirely manually, or through pushbutton override of traffic controller signal operation. When allocating rights-of-ways at intersections, the primary goal of traffic control officers is to manage extreme peak flow conditions and associated extensive traffic queues as efficiently and safely as possible. Intersection right-of-way allocation times are based solely on the judgment of traffic control officers and with vehicle queues extending for miles at critical bottleneck locations. Traffic signal cycle lengths resulting from traffic control officers can be extremely long. In the 1950 s, Webster conducted a series of experiments on pre-timed isolated intersection operations and found that for a simple two phased traffic signal that the relative increase in capacity for cycle lengths greater than
100 seconds is very small $[3,4]$. Routine observation of Clemson game day traffic at intersections controlled by traffic officers indicates that cycle lengths greater than 100 seconds are the norm rather than the exception. An obvious question to ask is "Are these longer cycle lengths necessary (or even beneficial)? This research focuses on answering this and other questions related to optimal traffic flow and traffic officer controlled intersections.

Specific goals of this research include: 1) an assessment of traffic officer effectiveness in operating signalized intersections during oversaturated conditions, 2) an evaluation of traffic officer performance to facilitate balanced queue dissipation at major intersections, 3) a comparison of measured traffic volumes, congestion, delay and vehicle queues to results determined from optimized computer modeled simulation, and 4) develop recommendations on how traffic officers can control intersections to optimally accommodate special event traffic flows.

\section{Literature Review}

Special events, such as college football games, cause overcrowding on primary routes, as drivers search for the fastest and easiest route to reach their destination. Spreading special event traffic over the entire network is the approach for reducing congestion. Event planners often distribute traffic onto alternate routes through use of pre-event publicity, dynamic message boards, enforcement, or road closures, according to Crawford [5]. Balancing network peak traffic flows for large special events draws from fundamental peak traffic flow parameters, the role of law enforcement in traffic control, and the comparison with simulation models. Research citations for each of these areas are provided in the following sections.

\subsection{Manual Traffic Control Operation}

Considerable research has focused on comparing manual signal control to that of the state-of-the practice traditional automatic signal control methods. For the purpose of this research, manual signal control refers to control of the intersection either by an officer's pushbutton at the controller cabinet, or by several officers standing within the intersection using hand gestures. Prior to the advent of automated traffic signals, traffic control officers were the only source of intersection traffic control. Historic research by Marsh compared advantages and disadvantages of manual control with automated signal control many decades ago, summarizing advantages of manual control as: 1) an officer has the ability to allocate time appropriately at any given instance; and 2) an officer can give priority to emergency and public transit vehicles [6]. Furthermore, Marsh determined an officer can handle varying left turn lane volumes better than automated signal control systems and can use beneficial judgment on a moment's notice [6]. Similar research by Parr found manual control of traffic signals can be an effective means to handle nonrecurring special event traffic [7]. Traffic control officers are able to use judgment to provide right-of- 
way and to clear queues. Parr concluded that officer effectiveness in directing traffic is a function of training and experience.

\subsection{Special Event Simulation}

Ding evaluated manual multi-modal signal control performance of traffic control agencies [8]. Performance of experienced traffic control agents (TCAs) was compared with optimal traffic signal control. An experiment using the manual intersection control simulator (MIC-Sim) was conducted to address three components: 1) a human, 2) a human-traffic control interface, and 3) a commercial traffic simulator. During the experiment, TCAs observed traffic conditions, such as the number of vehicles in queue, and manually controlled the traffic by clicking the corresponding traffic movement phases in the control panel displayed on the screen. For the same traffic volume, an online optimization tool was used to control traffic. A comparison of the two scenarios in terms of delay and throughput showed that optimal control outperformed manual control with a $29.2 \%$ reduction in delay and increased throughput [8].

Lassacher presented a variety of traffic management strategies to manage congestion resulting from football games at Montana State University [9]. During game-day traffic control, only one intersection required manual control where traffic control officers managed right-of-way assignment at the intersection of 11th Avenue and College Street, which is an all-way stop-controlled (AWSC) intersection under normal conditions. A simulation model of this intersection was created to compare manual operation with AWSC. Findings of this research showed game traffic operation benefited greatly from manual control. However, overall LOS and delay at the intersection still remained poor. Southbound traffic, which is primarily game traffic, experienced reduced control delay. All other approaches experienced increased control delay due to manual operation [9].

Using traffic data extracted from video feeds, Parr analyzed and modeled manual traffic control for signalized intersections at Louisiana State University home games in Baton Rouge, Louisiana [7]. The research developed a field logit model based on field observations to serve as the signal controller for the simulation model of study intersections. The logit model was developed based on weights given by officers controlling the traffic in the field, essentially mirroring manual operation of intersections in the simulation model. All of the intersections in the study area were operated by pushbutton manual control. Researchers compared pushbutton control with actuated signal control on various parameters such as average delay, average number of stops, average speed, average stopped delay, total delay, and total travel time. The findings indicated actuated signal control outperformed the manual control under every single metric. Manual control experienced a substantial decrease in saturation flow as phase length progressed due to random arrivals. The inability to skip phases in pushbutton control was identified as another reason for the poor performance of manual control.

A good summary of the use of simulation in the traffic management of special events can be found in Amini, et al [10].

\subsection{Relationship Between Capacity and Cycle Length}

In 1958, Webster developed an equation to determine the relationship between cycle length and critical traffic volume while considering signal phasing, loss time, and saturation flow rate headway [3]. Webster's equation for the maximum sum of critical volumes that can be accommodated for a cycle length is as follows:

$$
V_{c}=\frac{1}{h}\left[3600-N \times t_{L} \times\left(\frac{3600}{C}\right)\right]
$$

Where: $V_{c}$ is the critical volume.

$h$ is the headway.

$N$ is the total number phases in each cycle.

$t_{L}$ is the total loss time in each phase.

$\mathrm{C}$ is the total cycle length.

In reviewing variable relationships from this sentinel research, it can be observed that cycle length and critical volume are directly proportional to one another. An increase in cycle length will increase critical volume (essentially capacity), however, longer cycle lengths produce diminishing returns with regard to increased capacity.

Figure 1 depicts the relationship between capacity and cycle length for a simple two phase signal by solving Webster's equation for various cycle lengths assuming 5 seconds of loss time per phase. In Figure 1, capacity is plotted on the vertical axis and cycle length on the horizontal axis. For short cycle lengths up to 30 seconds, the relationship between capacity and cycle length is nearly linear with a steep upward slope. There is a continuous and significant change is slope between 30 and 60 seconds. The slope flattens considerably for cycle lengths greater than 60 seconds. An incremental increase in capacity for cycle lengths greater than 100 seconds is very small. The choice between longer and shorter cycle lengths is debatable. Longer cycle lengths result in increased capacity but also result in higher delays for movements that are not served. Shorter cycle lengths favor reduced delays on all approaches, but result in reduced capacity due to more loss time as percent of the cycle length. If volumes exceed capacity, cycle slips will occur and queues will increase rapidly, causing increased delays. Cascading network failures occur when vehicle queues disrupt the function of other downstream intersections, causing failures to extend backward through the surrounding roadway network. 


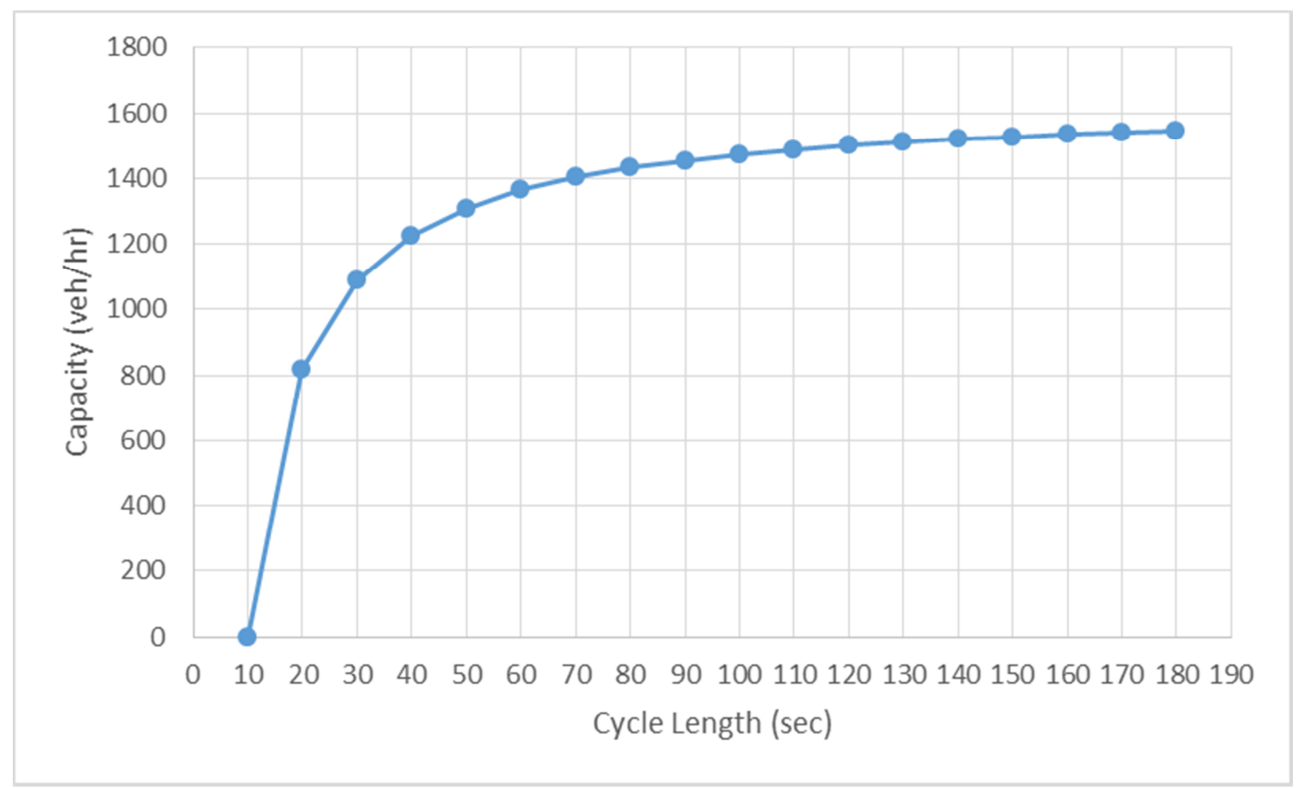

Figure 1. Relationship between Capacity and Cycle Length for a Two Phase Signal.

\section{Research Methodology}

The methodology includes data collection, data processing, model development, analysis, and comparison of findings. Game-day traffic data was collected for Louisville and South Carolina games during the 2014 football season, and for Notre Dame, and Florida State games during 2015 football season. Data was collected at seventeen critical network intersections on and around the Clemson University campus, see Figure 2 for a location map. Traffic control for these intersections is primarily performed by traffic officers, standing at intersections providing right-of-way distribution, using hand gestures. The reason for use of traffic officers is that on game-days these intersections reflect dramatic changes in geometry from their typical configuration including lane contraflow operations and/or prohibited movements. Another reason is that some locations are typically only two-way stop controlled intersections under normal operation but require a higher level of control on Clemson football game-days due to dramatically increased traffic. Traffic signalized intersections without geometric changes may continue to operate in a normal mode, or in pushbutton operation depending on demand.

In addition to the network optimization analysis, three critical intersections were identified for further analysis as a specific focus of this research: 1) College Avenue/SC-133 @ US-123/US-76; 2.) US-123@ @ US-76 and 3.) US-76@ Perimeter Road/S-39-320. These intersection locations were selected for detailed evaluation due to their level of congestion, geometry, control type, and traffic control officer responsibilities. These intersections use the two most common types of manual intersection control. College Avenue@ US-123 was controlled by police officers standing in the middle of the intersection and providing right-of-way using hand gestures. For the intersection at US-123@, US-76, an officer provided control using a cabinet pushbutton. Pushbutton techniques allow officers to control the signal indications by either extending a phase when needed or changing phases by manually clicking a button. The third intersection, US-76 @ Perimeter Road/S-39-320 was included in the analysis because of its unique geometry and level of congestion (T-intersection with a contraflowed approach and multiple turning lanes). This intersection was controlled by officers using hand gestures.

The data for selected intersections was collected, analyzed, and evaluated by faculty and graduate students in Clemson's Civil Engineering Department. Count volumes were obtained from video feeds and raw count data was adjusted based on queue data to determine estimated values for actual approach demand. A SYNCHRO model of the critical intersections operated manually by police officers using hand gestures or with pushbuttons was developed. Estimated approach demand volumes were used as input into the SYNCHRO model. Performance measures for right-of-way timings allocated by police officers were compared with performance measures for optimal signal timings obtained from SYNCHRO. Total delay for each approach was considered as the measure-of-effectiveness (MOE) in each condition. Since SYNCHRO is not a microscopic simulation model and as a result the delay model may not accurately reflect delay in oversaturated conditions, a calibrated VISSIM model of these three intersections was created to obtain vehicle delays at approaches and to observe queue estimation under manual signal control and optimized model conditions. Research results provide a comparison of these two models and provide additional insight into peak flow traffic control and the important role of law enforcement in implementing an effective special event plan. 


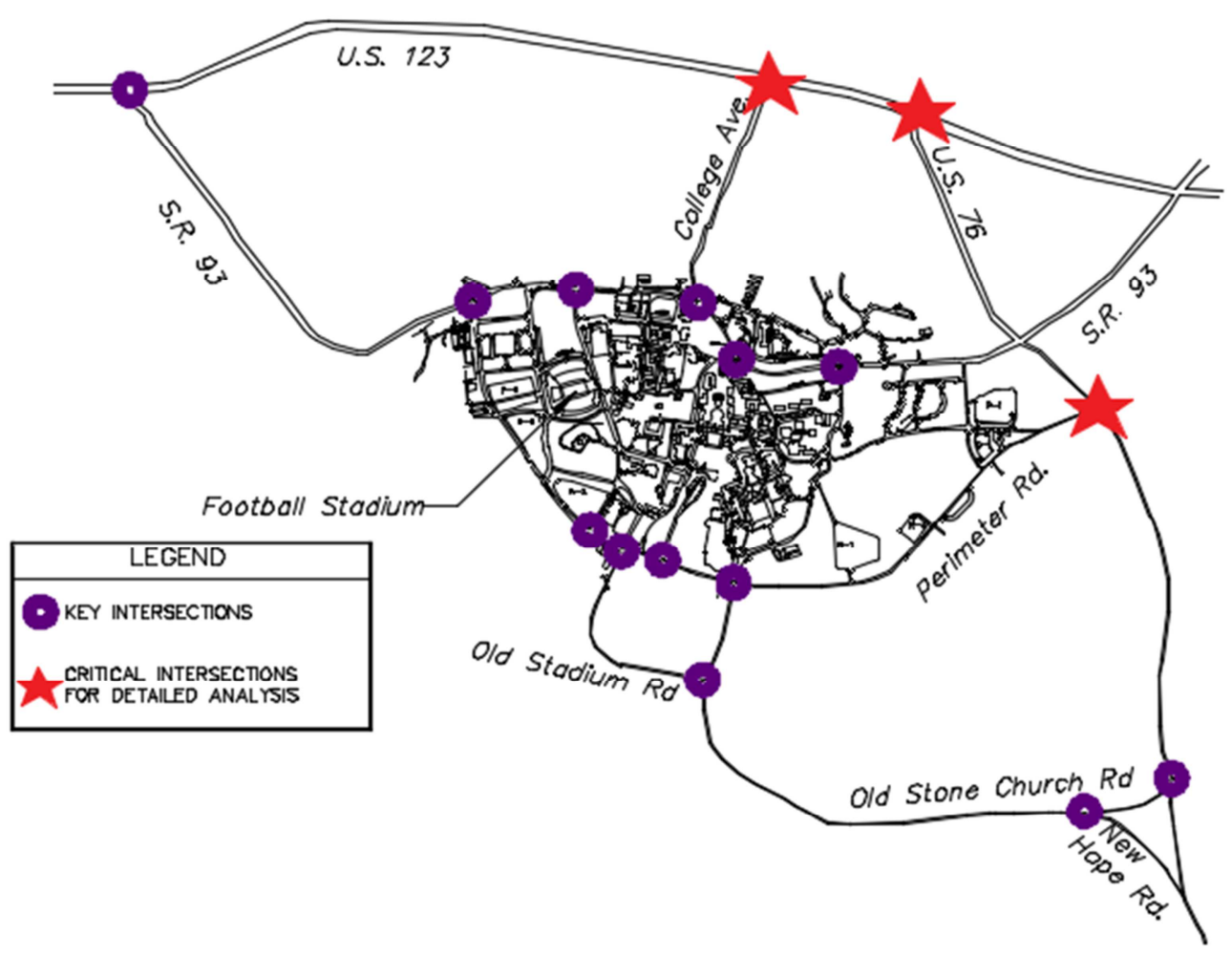

Figure 2. Study Area and Data Collection locations for Special Event Traffic.

\section{Simulation Findings and Results}

Existing manual operation of traffic signals and simulation model cycle lengths and vehicle delays are summarized for each of the three critical intersection locations for the highest peak flow periods occurring as indicated either before or after home football games.

\subsection{US-123@US-76 Intersection}

The analysis for the US-123@ US-76 focused on before game traffic because this intersection is the first major intersection that traffic coming on US-123 (westbound approach) has to pass through heading into town, towards the stadium. On football game days this intersection experiences no geometric changes and is controlled by pushbutton. The average equivalent cycle length used by traffic officers at this intersection is 174 seconds. For the same volume using the optimize cycle length and optimize splits features of SYNCHRO, the optimized cycle length was found to be 65 seconds. Differences in signal phase timings are summarized in Figure 3. As summarized in Table 1, research findings show that the optimized timings reduce delay on the northbound approach by $87 \%$. The southbound approach has little volume because it is a driveway from Ace Hardware. The westbound and eastbound approaches experienced an increase in delay as a result of optimized timings. Traffic officers were observed to allot more green time for the eastbound approach to provide greater priority to fans arriving in Clemson which was certainly reasonable to expect. Another finding from field observation was that the officers did a good job of clearing traffic queues on all intersection approaches.

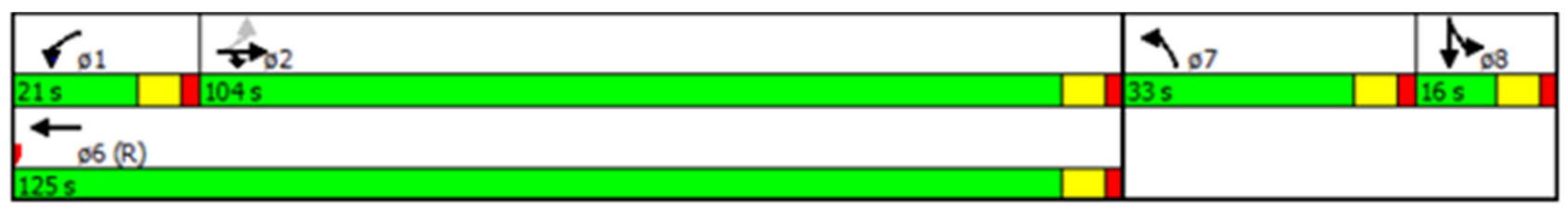

Manual Pushbutton Signal Operation Timings

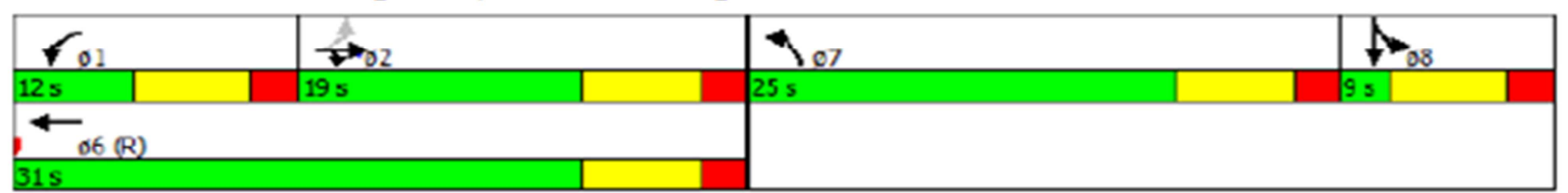

Optimized Model Signal Timings

Figure 3. Signal Timing Comparisons for US 76 @ US 123 Intersection, before game. 
Table 1. Approach Delay Comparisons for US $76 @$ US 123 Intersection, before game.

\begin{tabular}{|c|c|c|c|c|c|c|c|c|}
\hline \multirow{2}{*}{ Movement } & \multicolumn{4}{|l|}{ Eastbound } & \multicolumn{4}{|l|}{ Westbound } \\
\hline & EBT & & EBR & & WBL & & WBT & \\
\hline Type of Delay & Avg. Delay & Total Delay & Avg. Delay & Total Delay & Avg. Delay & Total Delay & Avg. Delay & Total. Delay \\
\hline Manual Operation & 19 & 836 & 1 & 27 & 71 & 639 & 6 & 606 \\
\hline Optimization Software & 24 & 1056 & 1 & 27 & 26 & 234 & 14 & 1414 \\
\hline$\%$ Improvement & -26 & -26 & 0 & 0 & 63 & 63 & -133 & -133 \\
\hline
\end{tabular}

Table 1. Continued.

\begin{tabular}{lllll}
\hline \multirow{2}{*}{ Movement } & Northbound & Southbound & \\
\cline { 2 - 5 } & NBL & & SB All & Total Delay \\
\hline Type of Delay & Avg. Delay & 2117 & Avg. Delay & 63 \\
\hline Manual Operation & 73 & 464 & 63 & 17 \\
Optimization Software & 16 & 78 & 17 & 73 \\
\% Improvement & 78 & 73 & \\
\hline
\end{tabular}

Movement average delays are provided in seconds/vehicle. Total delays are in seconds for a peak 5-minute period.

\subsection{College Avenue@US-123 Intersection}

Game day travel time studies indicate that this intersection experiences some of the worst delays throughout the network after football games conclude. One characteristic of aftergame traffic is that attendees try to leave at the same time, and the induced peak overwhelms the transportation network. The College Avenue@ US-123 intersection was analyzed with the data from the Clemson vs. Notre Dame Game played during the 2015 season. This game attracted a sellout crowd as well as ESPN College GameDay. Typically after games, the northbound and eastbound approaches at this intersection are the most heavily affected by game-related traffic. One important observation to note was that College Avenue was closed after the game at SC-93 to help reduce northbound traffic on College Avenue. From the videos taken, it was observed that the drivers still managed to cut through neighborhoods and enter College Avenue. This resulted in heavier-than-expected traffic on the intersection's northbound approach. Also, police officers rationed relatively more time to NB than EB even though travel time studies indicate that the EB approach experiences the longest queues and average delay per vehicle. The cycle length for manual hand signal operation at this intersection was found to be 509 seconds, and the optimized cycle length for the same demand volumes was found to be 205 seconds. Differences in signal phase timings are summarized in Figure 4. VISSIM Delay comparisons between manual hand signal operation and optimized operation for College Avenue @ US 123 are summarized in Table 2. Results from VISSIM indicate that the optimized timing results in fewer delays for all movements.

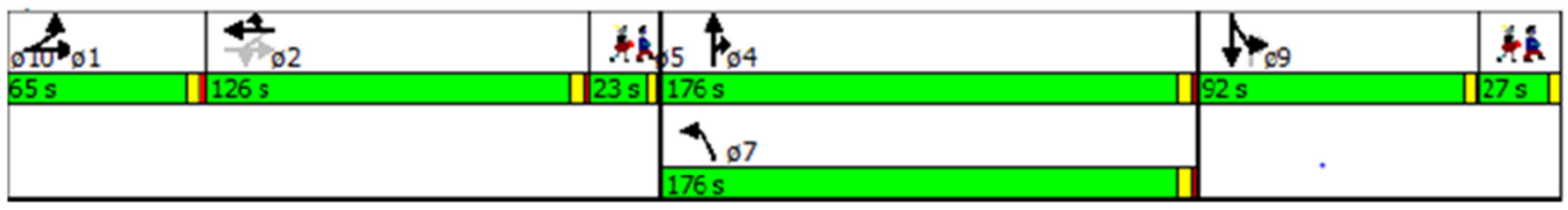

Manual Hand Signal Operation Timings

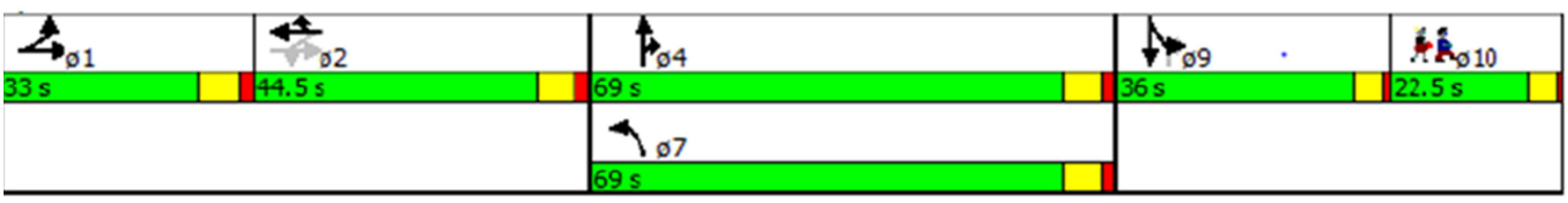

Optimized Model Signal Timings

Figure 4. Signal Timing Comparisons for CollegeAvenue@US 123, aftergame.

Table 2. Approach Delay Comparisons for College Avenue@US 123, after game.

\begin{tabular}{|c|c|c|c|c|c|c|c|c|c|c|}
\hline \multirow{2}{*}{ Movement } & \multicolumn{5}{|c|}{ Eastbound } & \multicolumn{5}{|c|}{ Northbound } \\
\hline & EBT & & EBL & & NBL & & NBT & & NBR & \\
\hline Type Delay & $\begin{array}{l}\text { Avg } \\
\text { Delay } \\
\end{array}$ & $\begin{array}{l}\text { Total } \\
\text { Delay } \\
\end{array}$ & $\begin{array}{l}\text { Avg } \\
\text { Delay } \\
\end{array}$ & $\begin{array}{l}\text { Total } \\
\text { Delay } \\
\end{array}$ & $\begin{array}{l}\text { Avg } \\
\text { Delay } \\
\end{array}$ & $\begin{array}{l}\text { Total } \\
\text { Delay } \\
\end{array}$ & $\begin{array}{l}\text { Avg } \\
\text { Delay } \\
\end{array}$ & $\begin{array}{l}\text { Total } \\
\text { Delay } \\
\end{array}$ & $\begin{array}{l}\text { Avg } \\
\text { Delay } \\
\end{array}$ & $\begin{array}{l}\text { Total } \\
\text { Delay } \\
\end{array}$ \\
\hline Manual Operation & 599 & 93444 & 763 & 21364 & 243 & 4617 & 247 & 8645 & 194 & 19206 \\
\hline Optimized Operation & 232 & 36192 & 308 & 8624 & 194 & 3686 & 210 & 7350 & 180 & 17820 \\
\hline \%Improved & 61 & 61 & 60 & 60 & 20 & 20 & 15 & 15 & 7 & 7 \\
\hline
\end{tabular}


Table 2. Continued

\begin{tabular}{|c|c|c|c|c|c|c|c|c|}
\hline \multirow{3}{*}{$\begin{array}{l}\text { Movement } \\
\text { Type Delay }\end{array}$} & \multicolumn{4}{|l|}{ Westbound } & \multicolumn{4}{|c|}{ Southbound } \\
\hline & WBT & & WBR & & SBL & & SBR & \\
\hline & Avg Delay & Total Delay & Avg Delay & Total Delay & Avg Delay & Total Delay & Avg Delay & Total Delay \\
\hline Manual Operation & 258 & 17544 & 235 & 4465 & 244 & 6344 & 133 & 3724 \\
\hline Optimized Operation & 139 & 9452 & 100 & 1900 & 174 & 4524 & 51 & 1428 \\
\hline \%Improved & 46 & 46 & 57 & 57 & 29 & 29 & 62 & 62 \\
\hline
\end{tabular}

Movement average delays are provided in seconds/vehicle. Total delays are in seconds for a peak 5-minute period.

\subsection{US76@Perimeter Road/SR-39-320}

Perimeter Road terminates at a T-intersection with US-76 at the east end of the Clemson campus. Perimeter Road is heavily affected by game day traffic produced by the parking lots it serves. US-76 is congested with traffic originating from SC-93 northbound via the on-ramp to US-76. This results in oversaturated conditions at the Perimeter-US 76 intersection. The cycle length for manual operation at this intersection was found to be 397 seconds, and the optimized cycle length for the same demand volumes was found to be 130 seconds. Differences in signal phase timings are summarized in Figure 5. Average and total delay comparisons between manual control and optimized control for US 76 @ Perimeter Road are summarized in Table 3. At this intersection optimized timing reduced average delay-pervehicle for all movements.

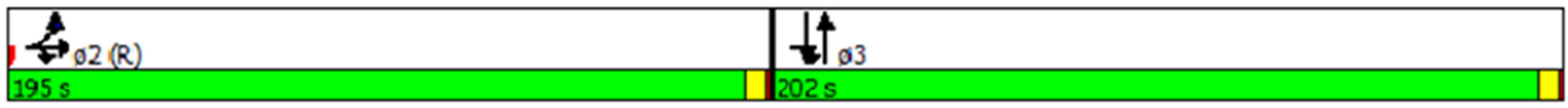

Manual Signal Operation Timings

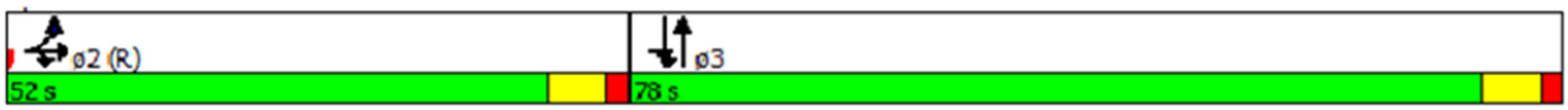

Optimized Model Signal Timings

Figure 5. Signal Timing Comparisons for US 76 @ Perimeter Road, after game.

Table 3. Approach Delay Comparisons for US76@Perimeter Road, after game.

\begin{tabular}{|c|c|c|c|c|c|c|c|}
\hline Movement & & NBT & & EBL & & EBR & \\
\hline Type Delay & Cycle Length & Avg. Delay & Total Delay & Avg. Delay & Total Delay & Avg. Delay & Total Delay \\
\hline Manual Control & 397 & 54 & 2430 & 420 & 18480 & 390 & 37830 \\
\hline Optimized Control & 130 & 18 & 810 & 397 & 17468 & 387 & 37539 \\
\hline
\end{tabular}

Table 3. Continued.

\begin{tabular}{|c|c|c|c|c|c|}
\hline \multirow{2}{*}{$\begin{array}{l}\text { Movement } \\
\text { Type Delay }\end{array}$} & \multirow[b]{2}{*}{ Cycle Length } & \multicolumn{2}{|l|}{ SBT } & \multicolumn{2}{|c|}{ SBT (from SC93 Ramp) } \\
\hline & & Avg. Delay & Total Delay & Avg. Delay & Total Delay \\
\hline Manual Control & 397 & 121 & 4840 & 214 & 15836 \\
\hline Optimized Control & 130 & 35 & 1400 & 41 & 3034 \\
\hline$\%$ Improved & & 71 & 71 & 81 & 81 \\
\hline
\end{tabular}

Movement average delays are provided in seconds/vehicle. Total delays are in seconds for a peak 5-minute period.

\section{Discussion and Conclusions}

A review of the analysis of the three selected intersections indicates that traffic officers performed best when intersections were not heavily oversaturated. Field observations of the two most heavily congested intersections confirm this finding. The officers consistently cleared queues on all approaches during the "ramp-up" period directly after the game. Once queues became sustained and oversaturated conditions developed, the officers' effectiveness diminished.

When comparing SYNCHRO optimized cycle length and timings with manual traffic officer control, it is important to note that there are two different objectives being followed. SYNCHRO algorithms attempt to minimize and balance delay for all approaches. Traffic officers often give priority to football patrons in an effort to clear long queues along the primary ingress and egress routes. For the manual pushbutton operated US-76@ US-123 before game scenario, SYNCHRO optimized timings were less efficient than the traffic officer's real-time judgement for the football traffic dominated movements. However the degree of saturation for this intersection was much less than the other two intersections.

There was little evidence that the two heavily oversaturated intersections benefited from traffic officer control as compared with optimized control. Model determined optimized timings reduced average delay-pervehicle for all movements - even the football dominated 
traffic movements that traffic officers most often give priority to. Use of extremely long cycle lengths by the officers only partially explains the disparity in delay comparisons between simulated manual and optimized control scenarios. A closer review of flow rates at 5-minute intervals indicates that vehicles tended to pass through the intersection at saturation headway during only an initial period. This flow rate tended to reduce below the theoretical saturation flow rate, even though the movement would appear to be saturated. An analysis of video recordings indicated a number of reasons for this phenomenon. For the US-123@ College Avenue intersection, the eastbound left-turn bay would back up excessively blocking one of the eastbound through lanes, which resulted in a considerably reduced through movement rate-of-flow. Vehicles entering and exiting at midblock driveways also contributed to increased vehicle headways. For the US-76@ Perimeter Road intersection, the dual leftturn lane from Perimeter Road would operate at the saturation flow rate of two lanes initially but the flow rate would reduce by one-half, once the initial left-turn lane (bay) vehicle queue cleared out.

\subsection{Implications for Special Event Traffic Planning}

Based on data collection, field observations, intersection operation, traffic simulations and research analysis, the following insights and conclusions, derived from Clemson University's experience with football game-day traffic operations, may be of interest to other jurisdictions using law enforcement officers in special event traffic operations are summarized as follows:

1. Traffic Control Officers display a tendency towards use of extremely long intersection cycle lengths during severely oversaturated traffic flow conditions, which may not be beneficial for any of the intersection approaches based on overall vehicle delay.

2. When intersections are significantly oversaturated, traffic control officers tend to have difficulty judging the extent of vehicles in excessively long queues, which often leads to some intersection approaches experiencing considerably disproportional adverse levels of vehicle delay.

3. Traffic Control Officers experience difficulty in detecting changes in flow rate that may lead to reduced efficiency in intersection operation and optimal allocation of right of way through phase durations. In many cases, officers readily identify the length of queues as vehicles visually extend back away from the intersection. This observation tends to result in prolonged manual phase timing extensions for backedup approaches, and as a consequence of longer phases corresponding vehicle flow rates occur that are considerably lower than desired demand-based saturation flow rates.

4. On average, traffic control officers using hand gestures typically produce greater lost-time than traffic-actuated signal operation for similar travel demand conditions occurring during special event peak traffic flows.
Intersections observed during Clemson football gameday traffic indicated that phase lost times range from 7 to 10 seconds when traffic officers use hand gestures to control intersections. This is nearly double typical signal phase lost times thus, shortening the cycle length will result in more cycles (and phases) per hour, which in turn will result in greater cumulative hourly lost-time. However during severely oversaturated conditions, this percent-increase in lost-time from a shorter cycle length may be offset by the efficiency gained through matching green time to the periods when saturation flow is present.

5. During periods of less severely saturated flow conditions, traffic control officers perform in a highly efficient manner using manual control methods in adapting to changing demand, reducing vehicle delay, and appropriately allocating phase times, especially when queue lengths and resulting dissipation can be readily observed from the officer's position.

\subsection{Recommendations for Traffic Control Officers}

Based on observations and findings from this research, additional recommendations on how traffic control officers can potentially improve special event traffic operations at crucial intersection locations are summarized as follows:

1. Traffic Control Officers should try to be cognizant in monitoring changes in traffic flow rates at controlled intersections. Relatively small observable reductions in approach vehicle rates of flow can be used as an indication that a phase change may be appropriate.

2. Traffic Control Officers should focus on identifying, detecting and understanding effects of reduction of vehicle approach flow rates and provide prompt reaction to adjustment of right of way allocation and timing sequences accordingly. One example may be to restart a left-turn phase to run concurrently with a priority through movement to ensure that queue length spillback interfering with optimal through movement flow does not occur.

3. Traffic Control Officers should strive to reduce vehicle delays on approaches experiencing special event-related traffic of highest demand, even if this results in increased delays on other intersection approaches.

4. Communication between traffic control officers at adjacent upstream and downstream intersections could improve detection of vehicle queue spillback problems and corresponding adverse impacts on different approaches, and provide better-optimized system level transportation network operation.

5. A data-driven approach is recommended for traffic control officer operation of crucial intersection locations. Oversaturated traffic conditions resulting from college football games and other similar special events are often recurring on a periodic basis, and thus, analysis of data from one event can be used to positively influence and improve network operations during subsequent special 
events.

6. Systematic, structured training programs and teambuilding debriefing and planning sessions should be included to address traffic flow and intersection operation topics identified in the list of recommendations identified above.

These findings and observations are further supported through numerous other special event traffic research citations discussed earlier in literature review of this paper. Thus, the authors believe these findings and recommendations should be of interest to other organizations and jurisdictions that are working to implement effective special event traffic plans that need to accommodate oversaturated traffic flow conditions.

\section{Acknowledgements}

The authors would like to acknowledge the Clemson University Athletic Department for supporting this work and the many law enforcement officials for their unwavering dedication to traffic safety and traffic operations management during the numerous on-campus special events held throughout every academic year.

\section{References}

[1] Deborah Matherly, Pamela Murray-Tuite, and Brian Wolshon. Traffic Management for Planned, Unplanned, and Emergency Events. In Traffic Engineering Handbook, John Wiley \& Sons, Inc, (2015) pp. 599-636.
[2] Latoski, S., W. Dunn, Jr., B. Wagenblast, J. Randall, M. Walker, Managing Travel for Planned Special Events, FHWAOP-04-010, Office of Transportation Management, Federal Highway Administration, U.S. Dept. of Transportation, Washington, DC, pp. 448, 2003.

[3] Webster, F. V., Traffic Signal Settings, Road Research Technical Paper No. 39, London, Her Majesty's Stationery Office, 1958: reprinted with minor amendments, 1969.

[4] Webster, F. V. and B. M. Cobbe, Traffic Signals, Technical Paper 56, Road Research Laboratory, London, 1966.

[5] Crawford, J., T. Carlson, W. Eisele, B. Kuhn, "A Michigan Toolbox for Mitigating Congestion," RC-1554, Texas Transportation Institute, College Sta., TX, pp. 278, 2011.

[6] W. Marsh, B. (1930). Traffic Signals, When and Where? National Safety Council.

[7] Parr, S. and B. Wolshon. "Methodology for Simulating Manual Traffic Control." Transportation Research Record, Journal of Transportation Research 2562 (2016): 9 - 17.

[8] Ding, N., Q. He, C. Wu, Performance Measurs of Manual Multi-Modal Traffic Signal Control, Proceedings of the $93^{\text {rd }}$ Annual Transportation Research Board Meeting, Washington, DC, Jan. 2013.

[9] Lassacher, S., D. Veneziano, S. Albert, Z. Ye, Traffic Management of Special Events in Small Communities, Transportation Research Record: Journal of the Transportation Research Board 2099 (2009): 85-93.

[10] Amini, Sasan \& Papapanagiotou, Eftychios \& Busch, Fritz. Traffic Management for Major Events. In Digital Mobility Platforms and Ecosystems, Ch. 4, Technische Universität München, (2016) pp. 187-197. 